\title{
99m Tc-Sulfur Colloid Bone Marrow Scintigraphy in Diagnosis of Diffuse Pulmonary Extramedullary Hematopoiesis Secondary to Myelofibrosis
}

\author{
Ming Yang ${ }^{1}$, Matthew F. Covington ${ }^{2}$, Ba D. Nguyen ${ }^{1}$, Geoffrey B. Johnson ${ }^{3}$, Ruben A. Mesa ${ }^{4}$, and Michael C. Roarke ${ }^{1}$ \\ ${ }^{1}$ Department of Radiology, Mayo Clinic in Arizona, Scottsdale, Arizona; ${ }^{2}$ Mallinckrodt Institute Radiology, Washington University \\ in St. Louis, St. Louis, Missouri; ${ }^{3}$ Department of Radiology, Mayo Clinic in Rochester, Rochester, Minnesota; and ${ }^{4}$ Division of \\ Hematology, Mays Cancer Center at UT Health San Antonio, MD Anderson Cancer Center, San Antonio, Texas
}

Our objective was to define the role of combined $99 \mathrm{mT}$ Tc-sulfur colloid bone marrow (SC BM) scintigraphy, SPECT or SPECT/ $\mathrm{CT}$, and chest CT in diagnosing diffuse pulmonary extramedullary hematopoiesis (PEMH) in patients with myelofibrosis. Methods: We retrospectively reviewed ${ }^{99 m T C-S C ~ B M ~ s c i n t i g r a-~}$ phy scans performed at our institution for the diagnosis of diffuse PEMH, as well as accompanying chest CT and SPECT/CT imaging findings. Relevant clinical information, including respiratory manifestations, pulmonary hypertension, and subjective response to whole-lung radiation therapy, was also summarized. Results: Twenty-two myelofibrosis patients with 27 99mTC-SC BM scintigraphy scans were diagnosed with diffuse PEMH. In 21 patients (95\%) with accompanying chest CT and SPECT/CT scans, the most common CT findings were groundglass opacity, interstitial infiltration, and pleural effusion. Of 20 patients $(91 \%)$ who underwent 2-dimensional echocardiography studies, 12 (55\%) were diagnosed with pulmonary hypertension. All 12 patients exhibited the aforementioned nonspecific CT imaging findings, with $8(66 \%)$ of them presenting with respiratory symptoms, including dyspnea, shortness of breath, and cough. In the remaining 8 patients, without pulmonary hypertension, half had similar respiratory symptoms. Fourteen patients (64\%) of this cohort received whole-lung radiation therapy, of whom 7 (50\%) experienced symptom relief after therapy. Conclusion: Nonspecific respiratory symptoms should raise concern about pulmonary hypertension and diffuse PEMH in patients with advanced-stage myelofibrosis. Combined 99mTc-SC BM scintigraphy and SPECT/ $\mathrm{CT}$ is a promising noninvasive imaging tool to diagnose this rare clinical entity.

Key Words: hematology; respiratory; SPECT/CT; pulmonary hematopoiesis; Tc-99m sulfur colloid scintigraphy; myelofibrosis

J Nucl Med Technol 2018; 46:368-372

DOI: 10.2967/jnmt.118.210534

Received Mar. 27, 2018; revision accepted May 31, 2018.

For correspondence contact: Ming Yang, Department of Radiology, Mayo Clinic in Arizona, 13400 E. Shea Blvd., Scottsdale, AZ 85259.

E-mail: yang.ming@mayo.edu

Published online Jun. 8, 2018

COPYRIGHT @ 2018 by the Society of Nuclear Medicine and Molecular Imaging.
$\mathbf{M}$ yelofibrosis is a chronic myeloproliferative bone marrow disease. It is characterized as a clonal disorder involving hematopoietic stem cells that causes ineffective erythropoiesis and dysplastic megakaryocyte hyperplasia (1). There are 2 forms of myelofibrosis: primary myelofibrosis, and myelofibrosis secondary to polycythemia vera or essential thrombocythemia. In the clinical course of myelofibrosis, as the bone marrow compartment becomes hyperfibrotic, extramedullary hematopoiesis $(\mathrm{EMH})$ ensues. EMH is the formation and development of blood cells outside the bone marrow, which is normal in fetal gestation but abnormal after birth. The most common sites of EMH are the liver and spleen, often resulting in hepatosplenomegaly. However, various other organs and tissues may also be involved, including the mediastinum, paravertebral regions, abdomen, pleural space, and lung, with associated site-specific symptoms and imaging manifestations (2).

In the thorax, EMH commonly occurs within the paravertebral regions and presents as a fatty density mass lesion on chest CT (3). Pulmonary EMH (PEMH) has also been sporadically described in the pleural space, pulmonary parenchyma, and even within the pulmonary artery $(4,5)$. The diffuse pattern of PEMH is uncommon and typically diagnosed at autopsy because of its nonspecific clinical manifestations (6-8). Clinically, diffuse PEMH usually presents with nonspecific respiratory symptoms and atypical CT imaging features (9-11). Sporadic case reports and small cohort studies suggest diffuse PEMH is causally associated with pulmonary hypertension.

${ }^{99 \mathrm{~m}} \mathrm{Tc}$-sulfur colloid bone marrow (SC BM) scintigraphy, a noninvasive nuclear medicine imaging modality, has been widely used for evaluation of bone marrow activity and diagnosis of EMH. This study reviewed the findings of ${ }^{99 \mathrm{~m} T c-S C}$ BM scintigraphy-in combination with chest CT and SPECT/CT - in myelofibrosis patients diagnosed with diffuse PEMH at a major tertiary-care hospital.

\section{MATERIALS AND METHODS}

We retrospectively reviewed the findings of ${ }^{99 \mathrm{~m}} \mathrm{Tc}-\mathrm{SC}$ BM scintigraphy performed on myelofibrosis patients at our institution from April 2009 to November 2016. Imaging findings from chest 


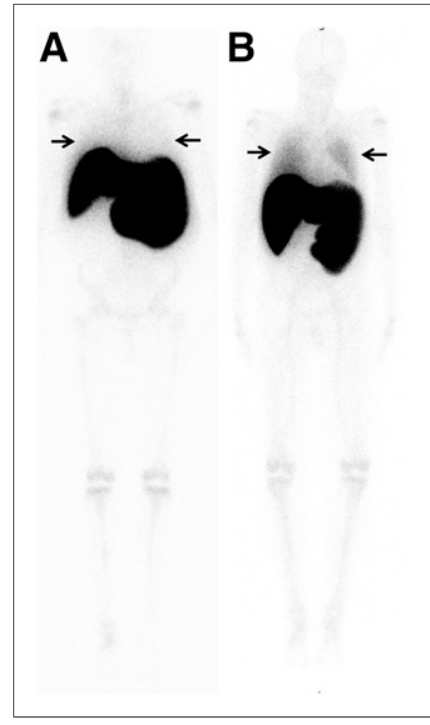

FIGURE 1. Whole-body planar $99 \mathrm{mT}$ T-SC BM scintigraphy of 2 patients with secondary myelofibrosis. (A) In patient without diffuse PEMH, uptake in both lung fields (arrows) is visually equivalent to that of blood pool. (B) In patient with diffuse $\mathrm{PEMH}$, uptake in lung fields (arrows) is greater than that of blood pool. Both patients exhibited $\mathrm{EMH}$ in liver and spleen, as well as decreased bone marrow uptake in central compartment of skeleton.

CT and accompanying SPECT/CT scans were also reviewed. Clinical charts were reviewed to obtain documented hemoglobin levels, clinical respiratory manifestations, 2-dimensional (2D) transthoracic echocardiography evidence of pulmonary hypertension, and patients' subjective responses to whole-lung radiation therapy. This study was approved by our institutional review board, and the requirement to obtain informed consent was waived.

\section{9mTc-SC BM Scintigraphic Imaging Protocol}

99m Tc-SC BM scintigraphy was performed at 2 nuclear medicine imaging laboratories at our institution. At site 1, an average dose of $370 \mathrm{MBq} \pm 10 \%$ of ${ }^{99 \mathrm{~m}} \mathrm{Tc}-\mathrm{SC}$ was administrated intravenously. Thirty minutes later, whole-body anterior and posterior planar images were simultaneously acquired with a dual-head camera. At site 2 , an average of $555 \mathrm{MBq} \pm 10 \%$ of ${ }^{99 \mathrm{~m} T c-S C}$ were administrated intravenously. Whole-body anterior and posterior planar images were acquired simultaneously with a dualhead camera with a 30- to 60-min delay. At both sites, a lowenergy, high-resolution collimator at $140 \mathrm{keV}$ with a $20 \%$ window was applied for planar imaging. If there was suggestive uptake in the lung fields, static anterior and posterior planar images of the chest were performed with the liver and spleen shielded, with the option of SPECT/CT of the chest.

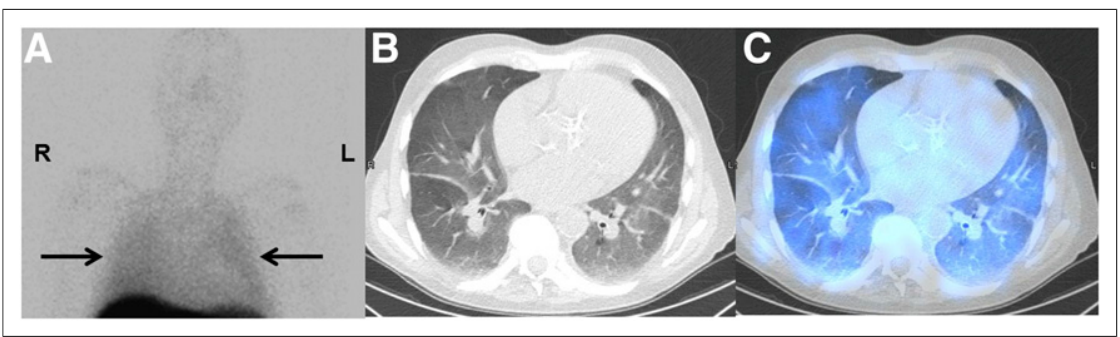

FIGURE 2. A 72-y-old man with end-stage myelofibrosis. (A) Planar imaging shows diffuse, mildly increased uptake in both lung fields (arrows), compared with blood pool. (B) Low-dose chest CT shows bilateral diffuse GGO at level of lower lobes. (C) Fused SPECT/CT at same level shows diffusely increased uptake, corresponding to findings on planar imaging. Patient died 5 mo later, after receiving whole-lung radiation therapy.

\section{Diagnostic Criteria}

A diagnosis of diffuse PEMH was made if the following criteria were met: EMH in the liver and spleen on ${ }^{99 \mathrm{~m}} \mathrm{Tc}-\mathrm{SC}$ BM scintigraphy planar images; diffusely increased uptake in both lung fields, visually greater in intensity than uptake in the blood pool; and no other focus of abnormally increased uptake in the thorax on combined SPECT/CT or chest CT images to indicate focal EMH involvement (Fig. 1).

On the basis of these criteria, we identified 22 myelofibrosis patients (of 49 patients) with $27{ }^{99 \mathrm{~m}} \mathrm{Tc}-\mathrm{SC}$ BM scintigraphy scans. Two independent observers certified by the American Board of Radiology and the American Board of Nuclear Medicine reviewed the ${ }^{99 m}$ Tc-SC BM scintigraphy images, chest CT images, and SPECT/CT images. Any differences in opinion were resolved by consensus.

Because no fine-needle aspiration or open lung biopsy was performed in this group of patients, confirmation of a diagnosis of diffuse PEMH was alternatively based on imaging findings of ${ }^{99 m}$ Tc-SC BM scintigraphy and clinical response to radiation therapy.

\section{RESULTS}

The 22 patients diagnosed with diffuse PEMH had an average age of $67 \pm 2.8 \mathrm{y}$ and a male-to-female ratio of 17:5. All 22 patients had had advanced-stage myelofibrosis for more than $3 \mathrm{y}$. The average hemoglobin level was $9.3 \mathrm{~g} / \mathrm{dL}$. Nine patients $(41 \%)$ had undergone splenectomy.

\section{CT Findings}

Twenty-one patients (95\%) had undergone diagnostic chest CT, and 3 (14\%) had also undergone SPECT/CT of the chest. On the images, the most common chest findings were one or more areas of ground-glass opacity (GGO) (13 patients, 62\%), interstitial infiltration (4 patients, 19\%), pleural effusion (4 patients, 19\%), and incomplete patchy atelectasis (2 patients, $9 \%$ ). No patients showed evidence of focal EMH in the pleural space, paravertebral region, or any other region in the thorax.

\section{Pulmonary Hypertension}

Twenty patients underwent transthoracic 2D Doppler echocardiography. Twelve patients (55\%) showed evidence of pulmonary hypertension, with a systolic pressure greater than $25 \mathrm{~mm} \mathrm{Hg}$. Additional ancillary echocardiography findings consisted of various degrees of right ventricle dilation or tricuspid valve regurgitation. All 12 patients had chest CT images available, of which $9(75 \%)$ exhibited GGO, 2 exhibited small pleural effusions, and 1 exhibited diffuse pulmonary infiltration (Figs. 2 and 3). There were 8 patients $(67 \%)$ without echocardiography evidence of pulmonary hypertension. Among them, 4 (50\%) exhibited GGO on chest CT, 2 demonstrated infiltrative changes, 1 had no pulmonary abnormalities, and 1 had no 


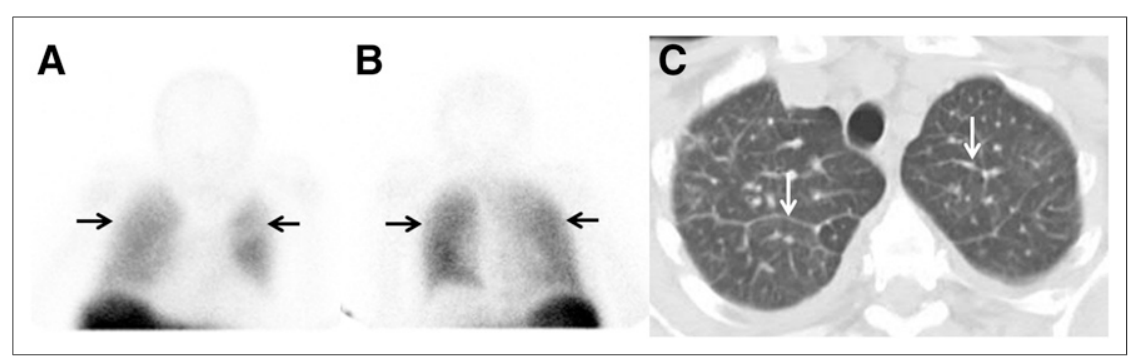

FIGURE 3. A 59-y-old woman with myelofibrosis after splenectomy. (A and B) Anterior $(A)$ and posterior $(B)$ thoracic static scintigraphy with partially shielded liver shows diffusely increased uptake in both lungs (arrows), compared with blood pool. (C) Chest CT shows diffuse, smooth interstitial thickening (arrows).

accompanying chest CT scan. In 2 patients without echocardiography studies, there was both pleural effusion and pulmonary infiltration on chest CT. The relationship between pulmonary hypertension and CT findings was summarized in Table 1.

\section{Respiratory Manifestations}

Of the 12 patients with echocardiography evidence of pulmonary hypertension, $8(66 \%)$ presented with dyspnea, shortness of breath, and cough, whereas of the 8 patients without echocardiography evidence of pulmonary hypertension, only $4(50 \%)$ presented with a similar clinical manifestation. In 2 patients without a $2 \mathrm{D}$ transthoracic echocardiography study, 1 patient $(50 \%)$ had a cough. The distribution between pulmonary hypertension and respiratory manifestations was summarized in Table 2.

\section{Whole-Lung Radiation Therapy}

Fourteen patients (64\%) in this cohort underwent wholelung, high-energy photon radiation beam therapy with the anteroposterior-posteroanterior technique up to a maximal dose of 100 cGy in a single fraction. Seven patients (50\%) experienced subjective relief or improvement of respiratory discomfort after radiation therapy.

Interval development or progression of diffuse PEMH was identified in 4 of 22 patients (18\%) with follow-up ${ }^{99 \mathrm{~m} T c-S C ~ B M}$ scintigraphy studies. One of these patients had decreased tracer uptake in the lung after radiation therapy and showed bilateral recurrence of diffuse PEMH on a follow-up scan (Fig. 4).

TABLE 1

Pulmonary Hypertension Versus CT Findings

\begin{tabular}{lcccc}
\hline CT findings & $\begin{array}{c}\mathrm{PH} \\
\text { present }\end{array}$ & $\begin{array}{c}\mathrm{PH} \\
\text { absent }\end{array}$ & $\begin{array}{c}\text { Echo findings } \\
\text { absent }\end{array}$ & Total \\
\hline Present & 12 & 7 & 2 & 21 \\
Absent & 0 & 1 & 0 & 1 \\
Total & 12 & 8 & 2 & 22 \\
& & & & \\
\hline $\mathrm{PH}=$ pulmonary hypertension; echo = echocardiography.
\end{tabular}

At the end of the study, 18 patients (83\%) had died 3 to 36 mo after diagnosis of diffuse PEMH, including 7 patients (39\%) who received wholelung radiation therapy during their clinical course.

\section{DISCUSSION}

EMH refers to hematopoiesis taking place outside the bone marrow compartment. It is a common complication in multiple hematologic diseases, including myelofibrosis, sickle cell anemia, and thalassemia. It occurs mainly in the liver and spleen, typically manifesting as hepatosplenomegaly. However, EMH may also occur in various other organs or structures, which are categorized as nonhepatosplenic EMH (2), of which prior splenectomy may be an etiologic factor. The spleen plays an important role in the removal of displaced hematopoietic stem cells. If the spleen is resected, hematopoietic stem cells may deposit at other sites (2). In our cohort, the fact that 9 patients (41\%) had prior splenectomy might support this theory. The clinical indications for scintigraphy imaging to evaluate EMH are to determine the extent of EMH in the liver or spleen, to evaluate for splenectomy, and to differentiate between EMH and other processes for any suggestive mass-like lesions outside the liver and spleen (12).

${ }^{99 \mathrm{~m}} \mathrm{Tc}-\mathrm{SC}$ BM scintigraphy is a well-accepted, noninvasive nuclear medicine imaging modality to evaluate bone marrow activity by targeting the reticuloendothelial system, which consists of macrophages located mainly in the liver, spleen, and bone marrow. After intravenous injection, radiolabeled colloids are phagocytosed by macrophages throughout the reticuloendothelial system. Because reticuloendothelial system and erythropoietic tissues coexist in the bone marrow, the in vivo ${ }^{99 \mathrm{~m}} \mathrm{Tc}-\mathrm{SC}$ distribution is an indicator of erythropoietic activity $(13,14)$. SPECT/CT images from 3 patients in our study provided additional value by confirming the absence of focal increased uptake in the pleural space and paraspinal regions (Fig. 2), thereby confirming the diagnosis of diffuse PEMH.

Our study also showed that common CT features of diffuse PEMH include nonspecific diffuse GGO, pulmonary

TABLE 2

Pulmonary Hypertension Versus Respiratory Symptoms

\begin{tabular}{lcccc}
\hline $\begin{array}{c}\text { Respiratory } \\
\text { symptoms }\end{array}$ & $\begin{array}{c}\mathrm{PH} \\
\text { present }\end{array}$ & $\begin{array}{c}\mathrm{PH} \\
\text { absent }\end{array}$ & $\begin{array}{c}\text { Echo findings } \\
\text { absent }\end{array}$ & Total \\
\hline Present & 8 & 4 & 1 & 13 \\
Absent & 4 & 4 & 1 & 9 \\
Total & 12 & 8 & 2 & 22 \\
\hline
\end{tabular}

$\mathrm{PH}=$ pulmonary hypertension; echo = echocardiography. 


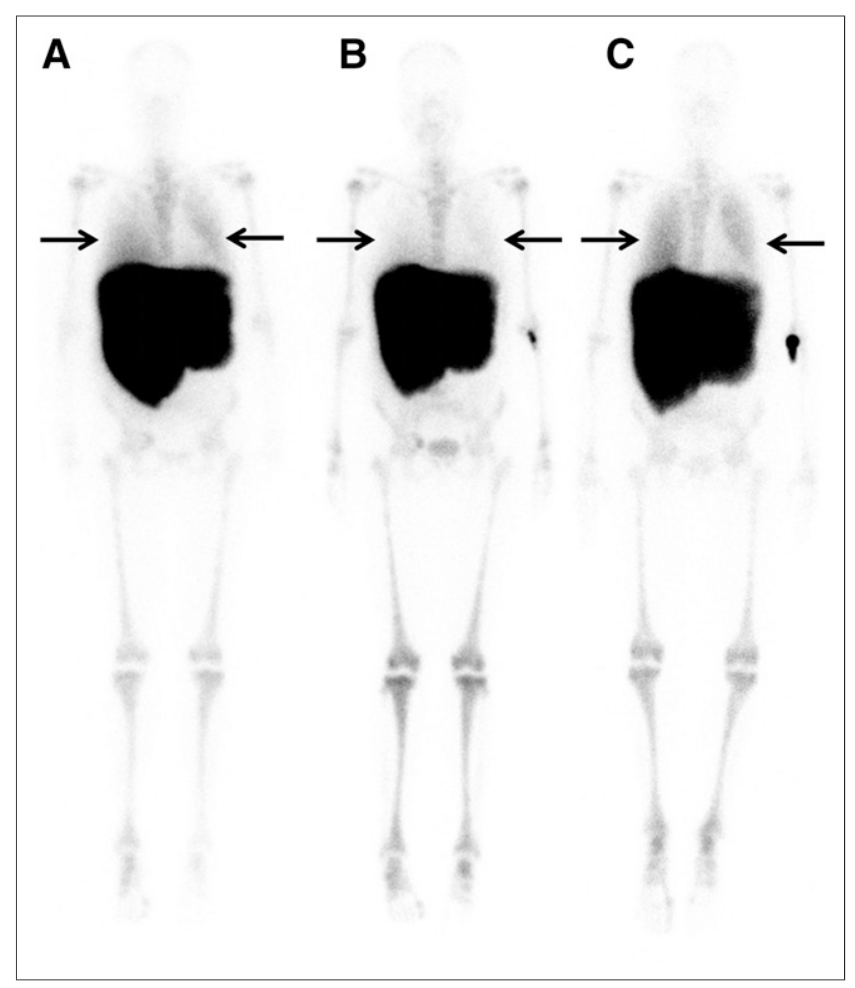

FIGURE 4. A 54-y-old female myelofibrosis patient with diffuse $\mathrm{PEMH}$ who underwent splenectomy and whole-lung radiation therapy, with additional radiation to liver. (A) Scintigraphy before radiation therapy shows diffusely increased uptake in both lung fields (arrows), compared with blood pool. (B) Two months later, near-complete resolution of pulmonary tracer uptake is seen (arrows), with accompanying substantial relief of respiratory discomfort. (C) Eleven months later, increased uptake in both lung fields reappears (arrows), suggesting recurrence of diffuse $\mathrm{PEMH}$.

nodules, or interstitial thickening, supporting the findings of prior studies $(2,6,15)$. A GGO is a nonspecific finding and may be present in heart failure, infection, chronic interstitial disease, and acute alveolar disease. However, in the clinical setting of myelofibrosis, a GGO should raise suspicion about the possibility of diffuse PEMH, thromboembolism, or chemotherapy-induced lung toxicity (16).

The most frequent clinical complaint in PEMH is dyspnea $(17,18)$, which might be caused by confounding chronic anemia, hypoxemia, hepatosplenomegaly, or pulmonary hypertension. Other less common etiologies for PEMH may also exist, including liver and bone marrow transplant, hepatic venoocclusive disease, liver cirrhosis, or liver metastasis $(16,19)$. None of these less common etiologies for PEMH were identified in our cohort of patients.

The association between pulmonary hypertension and myelofibrosis has been described in case reports and a small number of cohort studies (20-23). Pulmonary hypertension is defined as elevated systolic pulmonary pressure greater than $30 \mathrm{~mm} \mathrm{Hg}$. The exact etiology of pulmonary hypertension in myelofibrosis is unclear and might be multifactorial (24). Two major forms of pulmonary hypertension have been described in patients with a myeloproliferative disorder: pulmonary hypertension secondary to chronic thromboembolism and precapillary pulmonary hypertension, with the most frequent clinical manifestations being dyspnea, chest pain, and syncope (25). Noninvasive 2D Doppler echocardiography should be recommended to evaluate pulmonary hypertension, right ventricular dilation, and cardiac valvular disease in myelofibrosis patients with the aforementioned clinical manifestations.

Under microscopy, there is an accumulation of monocytes, megakaryocytes, and erythroblasts, with an associated obliteration of lymphatic flow, in specimens of PEMH (26). These microscopic findings are typical of myeloid metaplasia, which is sometimes used as an interchangeable term to describe the pathologic process of PEMH.

Hematopoietic tissues are extremely radiosensitive. Hence, the consensus treatment of diffuse PEMH is whole-lung, high-energy, low-dose radiation therapy (100 cGy at the anteroposterior and posteroranterior projections) $(27,28)$, which is usually curative without concern about radiation-induced myelotoxicity. In our cohort, half the treated cases (7/14) reported relief or improvement of clinical symptoms after radiotherapy.

Our study had several limitations. First, no lung biopsies were performed in this cohort of patients to provide pathologic confirmation of PEMH out of concern about a potential sampling error and risk of pulmonary hemorrhage, particularly in the setting of pulmonary hypertension. However, on the basis of our institutional experience, imaging with 99m Tc-SC BM scintigraphy, SPECT/CT, or chest CT provides an accurate, noninvasive diagnosis of diffuse PEMH and, consequently, favorable outcomes in the management of myelofibrosis patients. Therefore, noninvasive imagingbased diagnosis of PEMH is likely preferable to percutaneous fine-needle aspiration or open lung biopsy in this setting.

Second, the binary diagnosis of diffuse PEMH in this retrospective study depended on visual inspection of uptake intensity in the left and right lung fields; a quantitative cutoff ratio of lung to blood pool is likely not necessary to confidently make this diagnosis on imaging. Additionally, it was not possible to perform a quantitative analysis on snapshot planar images in our retrospective review. Future studies using a quantitative cutoff ratio of lung to blood pool to diagnose PEMH could be of benefit.

Finally, among 14 patients who were treated with wholelung radiation therapy, only 1 had follow-up ${ }^{99 \mathrm{~m}} \mathrm{Tc}-\mathrm{SC}$ BM scintigraphy demonstrating resolution of diffuse PEMH in both lung fields (Fig. 4). Follow-up ${ }^{99 \mathrm{~m} T c-S C ~ B M}$ scintigraphy could potentially provide a noninvasive alternative to biopsy to confirm response to therapy, although such a practice cannot be supported given the single patient with both histopathologic and imaging follow-up.

\section{CONCLUSION}

Diffuse PEMH is a rare clinical entity, commonly seen in advanced-stage myelofibrosis patients presenting with nonspecific respiratory symptoms. We recommend ${ }^{99 \mathrm{~m}} \mathrm{Tc}-\mathrm{SC}$ BM 
scintigraphy with SPECT/CT as the noninvasive imaging modality of choice in the diagnosis of diffuse PEMH. In patients diagnosed with diffuse PEMH, prompt palliative whole-lung, low-dose radiation therapy should be initiated to alleviate respiratory symptoms and improve quality of life.

\section{DISCLOSURE}

No potential conflict of interest relevant to this article was reported.

\section{REFERENCES}

1. Lieberman PH, Rosvoll RV, Ley AB. Extramedullary myeloid tumors in primary myelofibrosis. Cancer. 1965;18:727-736.

2. Koch CA, Li CY, Mesa RA, Tefferi A. Nonhepatosplenic extramedullary hematopoiesis: associated diseases, pathology, clinical course, and treatment. Mayo Clin Proc. 2003;78:1223-1233.

3. Georgiades CS, Neyman EG, Francis IR, Sneider MB, Fishman EK. Typical and atypical presentations of extramedullary hemopoiesis. AJR. 2002;179:12391243.

4. Marchiori E, Escuissato DL, Irion KL, et al. Extramedullary hematopoiesis: findings on computed tomography scans of the chest in 6 patients. J Bras Pneumol. 2008;34:812-816

5. Monga V, Silverman M. Pulmonary extramedullary hematopoiesis involving the pulmonary artery. Hematol Rep. 2015;7:5714.

6. Asakura S, Colby TV. Agnogenic myeloid metaplasia with extramedullary hematopoiesis and fibrosis in the lung: report of two cases. Chest. 1994;105:18661868.

7. Ozbudak IH, Shilo K, Hale S, Aguilera NS, Galvin JR, Franks TJ. Alveolar airspace and pulmonary artery involvement by extramedullary hematopoiesis: a unique manifestation of myelofibrosis. Arch Pathol Lab Med. 2008;132:99103.

8. Pitcock JA, Reinhard EH, Justus BW, Mendelsohn RS. A clinical and pathological study of seventy cases of myelofibrosis. Ann Intern Med. 1962;57:73-84.

9. Ali SZ, Clarke MJ, Kannivelu A, Chinchure D, Srinivasan S. Extramedullary pulmonary hematopoiesis causing pulmonary hypertension and severe tricuspid regurgitation detected by technetium-99m sulfur colloid bone marrow scan and single-photon emission computed tomography/CT. Korean J Radiol. 2014;15:376380 .

10. Bowling MR, Cauthen CG, Perry CD, et al. Pulmonary extramedullary hematopoiesis. J Thorac Imaging. 2008;23:138-141.

11. Yang M, Roarke M. Diffuse pulmonary extramedullary hematopoiesis in myelofibrosis diagnosed with technetium-99m sulfur colloid bone marrow scintigraphy and single photon emission computerized tomography/CT. Am J Hematol. 2017;92:323-324.
12. Agool A, Glaudemans AW, Boersma HH, Dierckx RA, Vellenga E, Slart RH. Radionuclide imaging of bone marrow disorders. Eur J Nucl Med Mol Imaging. 2011;38:166-178.

13. Desai AG, Thakur ML. Radiopharmaceuticals for spleen and bone marrow studies. Semin Nucl Med. 1985;15:229-238.

14. Van Dyke D, Price D, Shkurkin C, Yano Y, Anger HO. Differences in distribution of erythropoietic and reticuloendothelial marrow in hematologic disease. Blood. 1967;30:364-374.

15. Trow TK, Argento AC, Rubinowitz AN, Decker RA. 71-year-old woman with myelofibrosis, hypoxemia, and pulmonary hypertension. Chest. 2010;138:15061510 .

16. Bajwa AA, Usman F, Wolfson D, Laos LF, Cury JDA. 62-year-old woman with dyspnea, leukocytosis, and diffuse ground-glass opacities. Chest. 2010;137:1470-1473.

17. Glew RH, Haese WH, McIntyre PA. Myeloid metaplasia with myelofibrosis: the clinical spectrum of extramedullary hematopoiesis and tumor formation. Johns Hopkins Med J. 1973;132:253-270.

18. Rumi E, Passamonti F, Boveri E, et al. Dyspnea secondary to pulmonary hematopoiesis as presenting symptom of myelofibrosis with myeloid metaplasia. Am J Hematol. 2006;81:124-127.

19. Jacobson AF, Marks MA, Kaplan WD. Increased lung uptake on technetium99m-sulfur colloid liver-spleen scans in patients with hepatic venoocclusive disease following bone marrow transplantation. J Nucl Med. 1990;31:372-374.

20. Dingli D, Utz JP, Krowka MJ, Oberg AL, Tefferi A. Unexplained pulmonary hypertension in chronic myeloproliferative disorders. Chest. 2001;120:801-808.

21. Garcia-Manero G, Schuster SJ, Patrick H, Martinez J. Pulmonary hypertension in patients with myelofibrosis secondary to myeloproliferative diseases. $\mathrm{Am} \mathrm{J} \mathrm{He-}$ matol. 1999;60:130-135.

22. Garypidou V, Vakalopoulou S, Dimitriadis D, Tziomalos K, Sfikas G, Perifanis V. Incidence of pulmonary hypertension in patients with chronic myeloproliferative disorders. Haematologica. 2004;89:245-246.

23. Perrone C, Cartolari R, Lupi B, Morelli S. Pulmonary hypertension diagnosed by echocardiography during idiopathic myelofibrosis: a case report and a brief review of the literature. Multidiscip Respir Med. 2010;5:267-270.

24. McLaughlin VV, Archer SL, Badesch DB, et al. ACCF/AHA 2009 expert consensus document on pulmonary hypertension: a report of the American College of Cardiology Foundation Task Force on Expert Consensus Documents and the American Heart Association developed in collaboration with the American College of Chest Physicians; American Thoracic Society, Inc.; and the Pulmonary Hypertension Association. J Am Coll Cardiol. 2009;53:1573-1619.

25. Kupferschmid JP, Shahian DM, Villanueva AG. Massive hemothorax associated with intrathoracic extramedullary hematopoiesis involving the pleura. Chest. 1993;103:974-975.

26. Kumar NB, Naylor B. Megakaryocytes in pleural and peritoneal fluids: prevalence, significance, morphology, and cytohistological correlation. J Clin Pathol. 1980;33:1153-1159.

27. Oren I, Goldman A, Haddad N, Azzam Z, Krivoy N, Alroy G. Ascites and pleural effusion secondary to extramedullary hematopoiesis. Am J Med Sci. 1999;318:286-288.

28. Weinschenker P, Kutner JM, Salvajoli JV, et al. Whole-pulmonary low-dose radiation therapy in agnogenic myeloid metaplasia with diffuse lung involvement. Am J Hematol. 2002;69:277-280. 